\title{
ПРИМЕНЕНИЕ МЕТОДА БУБНОВА-ГАЛЕРКИНА ДЛЯ ОПРЕДЕЛЕНИЯ СТАТИСТИЧЕСКИХ ХАРАКТЕРИСТИК СОСТОЯНИЯ ПРИЗМАТИЧЕСКОЙ ОБОЛОЧКИ
}

\author{
Кубаймуродов Даврон Ильясович \\ Научные руководители: Исмайилов Кубаймурат \\ доктор технических наук \\ Ибрагимов Абдижаббар \\ кандидат технических наук \\ Самаркандский Государственный \\ архитектурно-строительный институт
}

Аннотация: Применен метод В.3.Власова для расчета оболочки на случайно-неоднородном основании. Уравнения изгиба призматической оболочки без учета кручения и деформации контура приводятся к системе обыкновенных дифференциальных уравнений относительно нормального и тангенциального перемещений. Данная методика существенно отличается от метода малого параметра.

Ключевые слова: Призматической оболочки, контур, метода БубноваГалеркина, изгибная жесткость, математические ожидания, корреляционной функции, коэффициент постели.

\section{APPLICATION OF THE BUBNOV-GALERKIN METHOD FOR DETERMINING THE STATISTICAL CHARACTERISTICS OF THE STATE OF THE PRISMATIC SHELL}

Kubaymurodov Davron Ilyasovich Scientific advisers: Ibragimov Abdijabbar Ismayilov Kubaymurat

\begin{abstract}
V.Z.Vlasov method was applied to calculate the shell on a randomly inhomogeneous base. The equations of bending of the prismatic shell without taking into account torsion and deformation of the contour are reduced to a system of ordinary differential equations for normal and tangential displacements. This technique is significantly different from the small parameter method.

Key words: Prismatic sheath, contour, method of Bubnov-Galerkin, flexural rigidity, mathematical expectations, correlation function bed coefficient.
\end{abstract}


Введение. Исследование напряженно - деформированного состояния призматических оболочек коробчатого сечения при регулярном характере упругого основания обычно выполняется при помощи вариационного метода В.3.Власова [1]. Методика расчета подробно изложена в работе [2]. Этот подход основан на разложении перемещений в ряды по произведениям двух функций, зависящих от продольной и окружной координаты $\left(\begin{array}{llll}z & \multicolumn{1}{c}{\boldsymbol{c}} & \mathrm{c}\end{array}\right.$ соответственно). Применим метод В.3.Власова для решения ответствующей стохастической задачи, т.е. для расчета оболочки на случайно-неоднородном основании.

Методы. Как показано в статие [1], уравнения изгиба призматической оболочки без учета кручения и деформации контура приводятся к системе обыкновенных дифференциальных уравнений относительно нормального и тангенциального перемещений $V(z)$ и $U(z)$ :

$$
a U^{\prime \prime}-b U-b V=0 ; \quad b U^{\prime}+b V^{\prime \prime}-Q V=P .
$$

где обозначено:

$$
\left.\begin{array}{l}
a=\gamma a_{j k}=\frac{E}{G} \int_{s} \varphi_{j} \varphi_{k} \delta d s=\frac{E}{G}\left(d_{1}^{3} \delta_{1}+3 d_{2}^{3} \delta_{2}+d_{2} \delta^{3}\right), b=b_{j k}=\int_{s} \varphi_{j}^{\prime} \varphi_{k} \delta d s=2 d_{1} \delta_{1}, \\
Q=\frac{1}{G} \int c(z) \bar{\psi}(s) d s=d_{2} c(z) / G, \quad p=\frac{1}{G} \int_{s} p(z) \psi(s) d s=p(z) / G .
\end{array}\right\}
$$

В формулах (2) сохранены все обозначения предыдущего раздела кроме коэффициента $Q$, который здесь выражается через случайную функцию $c(z)$, характеризующую упругость стохастического основания. Этот коэффициент представляет работу реакций упругого основания, равномерно распределенных по плоскости контакта оболочки с основанием (рис.3.2).

Систему (1) можно привести к одному уравнению относительно разрешающей функции $F$, которая связана с искомыми перемещениями следующими зависимостями:

$$
U=b F^{\prime}, V=a F^{\prime \prime}-b F \text {. }
$$

Непосредственно подстановкой выражений (3) в уравнения (1) можно убедиться в том, что первое из этих уравнений удовлетворяется тождественно, а второе уравнение приводится к виду:

$$
F^{I V}-Q_{1}(z) F^{\prime \prime}+Q_{2}(z) F=-P ;
$$

где

$$
Q_{1}=\frac{Q}{b}=\frac{d_{2} c(z)}{G b} ; \quad Q_{2}=\frac{Q}{a}=\frac{d_{2} c(z)}{G a} ; \quad P=\frac{2 P(z)}{G a b} .
$$


Переходя к безразмерной переменной $\xi=z / \ell$, получаем

$$
F^{I V}-Q_{1}(\xi) \ell^{2} F^{\prime \prime}+Q_{2}(\xi) \ell^{4} F=-\ell^{4} P
$$

Таким образом, задача сводится к интегрированию уравнения. (5) с учетом того, что жесткость основания, характеризуемая функцией $c(z)$, меняется вдоль координаты $z$ случайным образом. Для того, чтобы приближенно решить эту задачу необходимо сделать дополнительные предложения о характере случайной функции $c(z)$. Как показано в работах $[2,3]$, удовлетворительные результаты можно получить, если принять, что функция $c(z)$ является стационарной в широком смысле и что ее ординаты распределены по нормальному закону. Стационарность функции $c(z)$ в широком смысле (или в смысле А.Я.Хинчина) означает, что математическое ожидание и дисперсия $c(z)$ постоянны, а корреляционная функция зависит только от разности $\Delta z=z_{2}-z_{1}$ и не зависит от самых значений $z_{1}$ и $z_{2}$. Исходя из вышеизложенного, для функции $c(z)$ примем следующие характеристики:

$$
\langle c(z)\rangle=c_{0}=\text { const } ; \quad D(c)=\sigma_{c}^{2}=\text { const } ; \quad K_{c}(\Delta z)=\sigma_{c}^{2} \cdot e^{-\beta|\Delta z|} .
$$

Разумеется, для корреляционной функции $K_{c}$ можно принять и какоелибо другое выражение, например

$$
K_{c}=\sigma_{c}^{2} \cdot e^{-\beta|\Delta z|}\left(\cos \gamma \Delta z+\frac{\beta}{\gamma} \sin \gamma|\Delta z|\right)
$$

имеющее участки с отрицательной корреляцией и соответствующе дифференцируемому случайному процессу. Однако, как показано во многих исследованиях, окончательные результаты мало зависят от вида корреляционной функции, а проведение необходимых выкладок, конечно, проще с функцией (6), чем с функцией (7)

Обыкновенное дифференциальное уравнение (5) имеет переменные коэффициенты $Q_{1}(\xi)$ и $Q_{2}(\xi)$, причем в отличие от рассмотренного детерминированного случая, эти коэффициенты являются стационарными случайными функциями координаты $\xi$. Приближенное решение будем строить при помощи обобщенного вариационного метода Бубнова-Галеркина, задав искомую функции $F(\xi)$ в виде:

$$
F(\xi)=A_{1} \psi_{1}(\xi)+A_{2} \psi_{2}(\xi) \text {. }
$$

Аппроксимирующие функции $\psi_{1}(\xi)$ и $\psi_{2}(\xi)$ выбираем с учетом граничных условий на свободных торцах оболочки при $\xi= \pm 1$

$$
M=E I \frac{\partial^{2} V}{\partial z^{2}}=0 ; \quad Q=E I \frac{\partial^{3} V}{\partial z^{3}}=0 ; \text { или } \quad \psi_{1}^{\prime \prime}=\psi_{1}^{\prime \prime \prime}=0 ; \quad \psi_{2}^{\prime \prime}=\psi_{2}^{\prime \prime \prime}=0 .
$$


Здесь $E I$ изгибная жесткость всего сечения оболочки.

В качестве аппроксимирующих функций $\psi_{1}(\xi)$ и $\psi_{2}(\xi)$ удобно использовать алгебраические полиномы следующего вида:

$$
\psi_{1}(\xi)=15+15 \xi^{2}-5 \xi^{4}+\xi^{6} ; \quad \psi_{2}(\xi)=1-\frac{407}{203} \xi^{2}+\frac{1083}{203} \xi^{4}-\frac{785}{203} \xi^{6}+\xi^{8} .(10)
$$

Заметим, что выражения для этих функций могут быть получены из выражений для функций $\psi_{1}(\xi)$ и $\psi_{2}(\xi)$, из предыдущего раздела, если для последних принять $\alpha=2$.

$\mathrm{B}$ обобщенном методе Бубнова-Галеркина коэффициенты $A_{1}$ и $A_{2}$ являются уже не постоянными числами, а случайными величинами. В качестве неизвестных выступают их вероятностные характеристики: математические ожидания, дисперсии, коэффициенты асимметрии, эксцесса и т.д. Именно в этом смысле применяемый вариационный метод назван обобщенным [3], основная же идея метода, вытекающая из принципа возможных перемещений, остается неизменной.

Применяя процедуру метода Бубнова-Галеркина к уравнению (5), получим

$$
\begin{aligned}
& \int_{0}^{1}\left[\left(\psi_{1}^{I V} A_{1}+\psi_{2}^{I V} A_{2}\right)-Q_{1}(\xi) \ell^{2}\left(\psi_{1}^{\prime \prime} A_{1}+\psi_{2}^{\prime \prime} A_{2}\right)+Q_{2}(\xi) \ell^{4}\left(\psi_{1} A_{1}+\psi_{2} A_{2}\right)+Q \ell^{4}\right] \psi_{1} \cdot d \xi=0 ; \\
& \int_{0}^{1}\left[\left(\psi_{1}^{I V} A_{1}+\psi_{2}^{I V} A_{2}\right)-Q_{1}(\xi) \ell^{2}\left(\psi_{1}^{\prime \prime} A_{1}+\psi_{2}^{\prime \prime} A_{2}\right)+Q_{2}(\xi) \ell^{4}\left(\psi_{1} A_{1}+\psi_{2} A_{2}\right)+Q \ell^{4}\right] \psi_{2} \cdot d \xi=0 .
\end{aligned}
$$

Введем следующие обозначения:

$$
\begin{aligned}
& a_{11}=\int_{0}^{1} \psi_{1}^{I V} \cdot \psi_{1} \cdot d \xi, \quad a_{12}=\int_{0}^{1} \psi_{2}^{I V} \cdot \psi_{1} \cdot d \xi, \quad a_{13}=\ell_{0}^{4} \int_{0}^{1} Q_{2}(\xi) \psi_{1}^{2} \cdot d \xi ; \\
& a_{14}=\ell_{1}^{1} \int_{0}^{1} Q_{2}(\xi) \psi_{1} \cdot \psi_{2} \cdot d \xi, \quad a_{15}=\ell^{2} \int_{0}^{1} Q(\xi) \psi_{1}^{\prime \prime} \cdot \psi_{1} \cdot d \xi ; \\
& a_{16}=\ell^{2} \int_{0}^{1} Q_{1}(\xi) \psi_{2}^{\prime \prime} \cdot \psi_{1} \cdot d \xi, \quad a_{17}=\ell^{4} P \int_{0}^{1} \psi_{1} \cdot d \xi ; \\
& b_{11}=\int_{0}^{1} \psi_{1}^{I V} \cdot \psi_{2} \cdot d \xi, \quad b_{12}=\int_{0}^{1} \psi_{2}^{I V} \cdot \psi_{2} \cdot d \xi, \quad b_{13}=\ell^{4} \int_{0}^{1} Q_{2}(\xi) \psi_{1} \psi_{2} \cdot d \xi ; \\
& b_{14}=\ell^{4} \int_{0}^{1} Q_{2}(\xi) \psi_{2}^{2} \cdot d \xi, \quad b_{15}=\ell^{2} \int_{0}^{1} Q_{1}(\xi) \psi_{1}^{\prime \prime} \cdot \psi_{2} \cdot d \xi ; \\
& b_{16}=\ell^{2} \int_{0}^{1} Q_{1}(\xi) \psi_{2}^{\prime \prime} \cdot \psi_{2} \cdot d \xi, \quad b_{17}=\ell^{4} P \int_{0}^{1} \psi_{2} \cdot d \xi .
\end{aligned}
$$

Система алгебраических уравнений (11) с учетом (12) запишется в виде $\left(a_{11}+a_{13}-a_{15}\right) A_{1}+\left(a_{12}+a_{14}-a_{16}\right) A_{2}+a_{17}=0$ $\left(b_{11}+b_{13}-b_{15}\right) A_{1}+\left(b_{12}+b_{14}-b_{16}\right) A_{2}+b_{17}=0$. 
Часть из этих коэффициентов являются постоянными числами:

$$
\begin{aligned}
& a_{11}=365,71 ; a_{12}=13,18 ; \quad a_{17}=19,14 \cdot P \cdot \ell^{4} ; \\
& b_{11}=13,18 ; \quad b_{12}=16,68 ; \quad b_{17}=0,957 \cdot P \cdot \ell^{4} ;
\end{aligned}
$$

Остальные коэффициенты представляют собой случайные величины:

$$
\begin{aligned}
& a_{13}=\ell^{4} \cdot \int_{0}^{1} Q_{2}(\xi) \psi_{1}^{2} d \xi=\frac{\ell^{4} d_{2}}{G a} \int_{0}^{1} c(\xi) \psi_{1}^{2} \cdot d \xi ; \quad a_{15}=\ell^{2} \cdot \int_{0}^{1} Q_{1}(\xi) \psi_{1}^{\prime \prime} \psi_{1} d \xi=\frac{\ell^{2} d_{2}}{G b} \int_{0}^{1} c(\xi) \psi^{\prime \prime} \cdot \psi_{1} d \xi ; \\
& a_{14}=\ell^{4} \int_{0}^{1} Q_{2}(\xi) \psi_{1} \cdot \psi_{2} d \xi=\frac{\ell^{4} d^{2}}{G a} \int_{0}^{1} c(\xi) \psi_{1} \psi_{2} d \xi ; \quad a_{16}=\ell^{2} \int_{0}^{1} Q_{1}(\xi) \psi_{2}^{\prime \prime} \cdot \psi_{1} d \xi=\frac{\ell^{4} d^{2}}{G b} \int_{0}^{1} c(\xi) \psi_{2}^{\prime \prime} \psi_{1} d \xi ; \\
& b_{13}=\ell^{4} \int_{0}^{1} Q_{2}(\xi) \psi_{1} \psi_{2} d \xi=\frac{\ell^{4} d_{2}}{G a} \int_{0}^{1} c(\xi) \psi_{1} \psi_{2} d \xi ; b_{15}=\ell^{2} \int_{0}^{1} Q_{1}(\xi) \psi_{1}^{\prime \prime} \cdot \psi_{2} d \xi=\frac{\ell^{2} d_{2}}{G b} \int_{0}^{1} c(\xi) \psi_{1}^{\prime \prime} \psi_{2} d \xi ; \\
& b_{14}=\ell^{4} \int_{0}^{1} Q_{2}(\xi) \psi_{2}^{2} d \xi=\frac{\ell^{4} d_{2}}{G a} \int_{0}^{1} c(\xi) \psi_{2}^{2} d \xi ; b_{15}=\ell^{2} \cdot \int_{0}^{1} Q_{1}(\xi) \psi_{2}^{\prime \prime} \psi_{2} d \xi=\frac{\ell^{2} d_{2}}{G b} \int_{0}^{1} c(\xi) \psi_{2}^{\prime \prime} \cdot \psi_{2} \cdot d \xi ;
\end{aligned}
$$

Таким образом, коэффициенты в уравнениях (13) представляют собой линейные комбинации постоянных и случайных величин. Для удобства дальнейшего анализа представим их в виде сумму средних значений и центрированных случайных величин

$$
a_{j}=\left\langle a_{j}\right\rangle+\tilde{a}_{j} ; \quad(j=1,2,3,4) .
$$

Здесь $\left\langle a_{j}\right\rangle$-математические ожидания коэффициентов $a_{j}, \tilde{a}_{j} \quad$-их флуктуации.

Коэффициенты $a_{j}$ связаны с введенными ранее величинами следующим образом:

$$
a_{1}=a_{11}+a_{13}-a_{15} ; \quad a_{2}=a_{12}+a_{14}-a_{16} ; \quad a_{3}=b_{11}+b_{13}-b_{15} ; \quad a_{4}=b_{12}+b_{14}-b_{16} .
$$

Математические ожидания коэффициентов (15) выражается через среднее значение коэффициента постели $c_{0}$ :

$$
\begin{aligned}
& \left\langle a_{1}\right\rangle=a_{11}+\left\langle a_{13}\right\rangle-\left\langle a_{15}\right\rangle=a_{11}+c_{0}\left(\frac{\ell^{4} d_{2}}{G a} \int_{0}^{1} \psi_{1}^{2} d \xi-\frac{\ell^{2} d_{2}}{G b} \int_{0}^{1} \psi_{1}^{\prime \prime} \cdot \psi_{1} \cdot d \xi\right) \\
& \left\langle a_{2}\right\rangle=a_{12}+\left\langle a_{14}\right\rangle-\left\langle a_{16}\right\rangle=a_{12}+c_{0}\left(\frac{\ell^{4} d_{2}}{G a} \int_{0}^{1} \psi_{1} \cdot \psi_{2} \cdot d \xi-\frac{\ell^{2} d_{2}}{G b} \int_{0}^{1} \psi_{2}^{\prime \prime} \cdot \psi_{1} \cdot d \xi\right) \\
& \left\langle a_{3}\right\rangle=b_{11}+\left\langle b_{13}\right\rangle-\left\langle b_{15}\right\rangle=b_{11}+c_{0}\left(\frac{\ell^{4} d_{2}}{G a} \int_{0}^{1} \psi_{1} \cdot \psi_{2} \cdot d \xi-\frac{\ell^{2} d_{2}}{G a} \int_{0}^{1} \psi_{1}^{\prime \prime} \cdot \psi_{2} \cdot d \xi\right) \\
& \left\langle a_{4}\right\rangle=b_{12}+\left\langle b_{14}\right\rangle-\left\langle b_{16}\right\rangle=b_{12}+c_{0}\left(\frac{\ell^{4} d_{2}}{G a} \int_{0}^{1} \psi_{2}^{2} d \xi-\frac{\ell^{2} d_{2}}{G b} \int_{0}^{1} \psi_{2}^{\prime \prime} \cdot \psi_{2} \cdot d \xi\right)
\end{aligned}
$$


Наряду с математическими ожиданиями случайных коэффициентов $a_{j}$ для выполнения статического анализа необходимы корреляционные моменты $\left\langle\tilde{a}_{j}, \tilde{a}_{k}\right\rangle$ Эти моменты выражаются через корреляционную функцию коэффициента постели $K_{c}\left(\xi-\xi^{\prime}\right)$

$$
K_{j k}=\left\langle\tilde{a}_{j}, \tilde{a}_{k}\right\rangle=\int_{0}^{1} \int_{0}^{1} K_{c}\left(\xi-\xi^{\prime}\right) C_{j k}\left(\xi, \xi^{\prime}\right) d \xi d \xi^{\prime} ;
$$

где $C_{j k}\left(\xi, \xi^{\prime}\right)$ есть произведение координатных функций, соответствующих методу Бубнова-Галеркина, формулы для $C_{j k}$ имеют вид:

$$
\begin{aligned}
& c_{11}=\left[\frac{\ell^{4} d_{2}}{G \cdot a} \psi_{1}^{2}(\xi)-\frac{\ell^{2} d_{2}}{G \cdot b} \psi_{1}^{\prime \prime}(\xi) \psi_{1}(\xi)\right]\left[\frac{\ell^{4} d_{2}}{G \cdot a} \psi_{1}^{2}\left(\xi^{\prime}\right)-\frac{\ell^{2} d_{2}}{G \cdot b} \psi_{1}^{\prime \prime}\left(\xi^{\prime}\right) \psi_{1}\left(\xi^{\prime}\right)\right] ; \\
& c_{12}=\left[\frac{\ell^{4} d_{2}}{G a} \psi_{1}^{2}(\xi)-\frac{\ell^{2} d_{2}}{G b} \psi_{1}^{\prime \prime}(\xi) \psi_{1}(\xi)\right]\left[\frac{\ell^{4} d_{2}}{G a} \psi_{1}\left(\xi^{\prime}\right) \psi_{2}\left(\xi^{\prime}\right)-\frac{\ell^{2} d_{2}}{G b} \psi_{2}^{\prime \prime}\left(\xi^{\prime}\right) \psi_{1}\left(\xi^{\prime}\right)\right] ; \\
& c_{21}=\left[\frac{\ell^{4} d_{2}}{G a} \psi_{1}(\xi) \psi_{2}(\xi)-\frac{\ell^{2} d_{2}}{G b} \psi_{2}^{\prime \prime}(\xi) \psi_{1}(\xi)\right]\left[\frac{\ell^{4} d_{2}}{G a} \psi_{1}^{2}\left(\xi^{\prime}\right)-\frac{\ell^{2} d_{2}}{G b} \psi_{1}^{\prime \prime}\left(\xi^{\prime}\right) \psi_{1}\left(\xi^{\prime}\right)\right] ; \\
& c_{22}=\ell\left[\frac{\ell^{4} d_{2}}{G a} \psi_{1}^{2}\left(\xi^{\prime}\right)-\frac{\ell^{2} d_{2}}{G b} \psi_{2}^{\prime \prime}(\xi) \psi_{1}(\xi)\right]\left[\frac{\ell^{4} d_{2}}{G a} \psi_{1}\left(\xi^{\prime}\right) \psi_{2}(\xi)-\frac{\ell^{2} d_{2}}{G b} \psi_{2}^{\prime \prime}\left(\xi^{\prime}\right) \psi_{1}\left(\xi^{\prime}\right)\right] .
\end{aligned}
$$

При $j=k$ формуле (17) дают значение дисперсий коэффициентов $a_{j}$.

Итак, система уравнений относительно коэффициентов разложения $A_{1}$ и $A_{2}$ принимает вид:

$$
\left(\left\langle a_{1}\right\rangle+\tilde{a}_{1}\right) A_{1}+\left(\left\langle a_{2}\right\rangle+\tilde{a}_{2}\right) A_{2}=b_{1} ; \quad\left(\left\langle a_{3}\right\rangle+\tilde{a}_{3}\right) A_{1}+\left(\left\langle a_{4}\right\rangle+\tilde{a}_{4}\right) A_{2}=b_{2} .
$$

где $b_{1}=-a_{17}, b_{2}=-b_{17}$.

Решение этих уравнений записывается следующим образом:

$$
\begin{aligned}
& A_{1}=\frac{b_{1}\left(\left\langle a_{4}\right\rangle+\tilde{a}_{4}\right)-b_{2}\left(\left\langle a_{2}\right\rangle+\tilde{a}_{2}\right)}{\left.\left(\left\langle a_{1}\right\rangle+\tilde{a}_{1}\right)\right)\left(\left\langle a_{4}\right\rangle+\tilde{a}_{4}\right)-\left(\left\langle a_{2}\right\rangle+\tilde{a}_{2}\right)\left(\left\langle a_{3}\right\rangle+\tilde{a}_{3}\right)} ; \\
& A_{2}=\frac{-b_{1}\left(\left\langle a_{3}\right\rangle+\tilde{a}_{3}\right)-b_{2}\left(\left\langle a_{1}\right\rangle+\tilde{a}_{1}\right)}{\left(\left\langle a_{1}\right\rangle+\tilde{a}_{1}\right)\left(\left\langle a_{4}\right\rangle+\tilde{a}_{4}\right)-\left(\left\langle a_{2}\right\rangle+\tilde{a}_{2}\right)\left(\left\langle a_{3}\right\rangle+\tilde{a}_{3}\right)} .
\end{aligned}
$$

Неизвестные случайные величины $A_{1}$ и $A_{2}$ на основании (20) явно выражаются через случайные аргументы $\tilde{a}_{1}, \tilde{a}_{2}, \tilde{a}_{3}, \tilde{a}_{4}$

$$
A_{1}=f_{1}\left(\tilde{a}_{1}, \tilde{a}_{2}, \tilde{a}_{3}, \tilde{a}_{4}\right), A_{2}=f_{2}\left(\tilde{a}_{1}, \tilde{a}_{2}, \tilde{a}_{3}, \tilde{a}_{4}\right) .
$$

Эти зависимости позволяют определить все статистические характеристики для $A_{1}, A_{2}$ по заданным характеристикам случайных величин $\tilde{a}_{j}$.

Допустим, что вектор $\tilde{a}\left\{\tilde{a}_{j}\right\}$ является гауссовским. Тогда его плотность вероятности записывается в следующей форме: 


$$
P(\tilde{a})=P\left(\tilde{a}_{1}, \tilde{a}_{2}, \tilde{a}_{3}, \tilde{a}_{4}\right)=\frac{1}{\sqrt{(2 \pi)^{4}|K|}} \exp \left(-\frac{1}{2} \sum_{j=1}^{4} \sum_{k=1}^{4}\left\{K^{-1}\right\} \tilde{a}_{j} \tilde{a}_{k}\right) .
$$

где $K\left\{K_{k j}\right\}$ есть корреляционная матрица (17), $|K|$ определитель матрицы $K,\left\{K^{-1}\right\}$ транспонированная матрица по отношению к $K$. Моменты случайных величин $A_{1}, A_{2}$ определяются как:

$$
\left\langle A_{m}^{n}\right\rangle=\int f_{m}^{n}(\tilde{a}) p(\tilde{a}) d \tilde{a} ;(m=1,2) ;
$$

где $p(\tilde{a})$ имеет вид $(21)$.

Выражение (22) дает принципиальное решение задачи. Однако его реализация затруднительна, поскольку сводится к вычислению четырехкратного интеграла. В практических расчетах моменты коэффициентов $A_{1}, A_{2}$, непосредственно выражаются через моменты величин $\tilde{a}_{j}$ на основании формул (20).

Представим зависимости (20) в виде разложения в ряд Тейлора:

$$
A_{m}=f_{m}^{(0)}+\frac{1}{1 !} \sum_{j=1}^{4} \frac{\partial f_{m}^{(0)}}{\partial \tilde{a}_{j}} \cdot \tilde{a}_{j}+\frac{1}{2 !} \sum_{j=1}^{4} \sum_{k=1}^{4} \frac{\partial^{2} f_{m}^{(0)}}{\partial \tilde{a}_{j} \partial \tilde{a}_{k}} \tilde{a}_{j} \tilde{a}_{k}+\ldots
$$

В разложении (23) обозначено:

$$
f_{m}^{(0)}=\int_{m}\left(\tilde{a}_{j}\right) \tilde{a}_{j=0} ; \quad \frac{\partial f_{m}^{(0)}}{\partial \tilde{a}_{j}}=\left.\frac{\partial f_{m}\left(\tilde{a}_{j}\right)}{\partial \tilde{a}_{j}}\right|_{\tilde{a}_{j}=0} .
$$

При практических расчетах соотношения (20) обычно линеаризуются, т.е. в ряду (23) удерживается члены не выше первой степени относительно $\tilde{a}_{j} \mathrm{~B}$ этом случае вычисления становится элементарными:

$$
\begin{aligned}
& \left\langle A_{m}\right\rangle=f_{m}^{(0)} . \\
& \left\langle A_{m} A_{l}\right\rangle=f_{m}^{(0)} f_{l}^{(0)}+\frac{1}{1 !} \sum_{j=1}^{4} \sum_{k=1}^{4} \frac{\partial f_{m}^{(0)}}{\partial \tilde{a}_{j}} \frac{\partial f_{l}^{(0)}}{\partial \tilde{a}_{k}}\left\langle\tilde{a}_{j} \tilde{a}_{k}\right\rangle \text { и т.Д. }
\end{aligned}
$$

Формулы (26) выведены на основе линеаризованного соотношения (23) и по существу соответствуют нулевому приближению метода малого параметра. Следующее приближение получается при удержании в (23) членов до второго порядка включительно:

$$
\left\langle A_{m}\right\rangle=f_{m}^{(0)}+\frac{1}{2 !} \sum_{j=1}^{4} \sum_{k=1}^{4} \frac{\partial^{2} f_{m}^{(0)}}{\partial \tilde{a}_{j} \partial \tilde{a}_{k}}\left\langle\tilde{a}_{j} \tilde{a}_{k}\right\rangle .
$$




$$
\begin{aligned}
& \left\langle A_{m} A_{l}\right\rangle=f_{m}^{(0)} f_{l}^{(0)}+\sum \sum\left(\frac{1}{1 !} \frac{\partial f_{m}^{(0)}}{\partial \tilde{a}_{j}} \frac{\partial f_{l}^{(0)}}{\partial \tilde{a}_{k}}+\frac{f_{m}}{2 !} \frac{\partial f_{l}^{(0)}}{\partial \tilde{a}_{j} \partial \tilde{a}_{k}}\right) \times \\
& \times\left\langle\tilde{a}_{j} \tilde{a}_{k}\right\rangle+\frac{1}{(2 !)^{2}} \sum_{j=1}^{4} \sum_{k=1}^{4} \sum_{r=1}^{4} \sum_{q=1}^{4} \frac{\partial^{2} f_{m}^{(0)}}{\partial \tilde{a}_{j} \partial \tilde{a}_{k}} \frac{\partial^{2} f_{n}^{(0)}}{\partial \tilde{a}_{j} \partial \tilde{a}_{k}}\left\langle\tilde{a}_{j} \tilde{a}_{k} \tilde{a}_{r} \tilde{a}_{q}\right\rangle .
\end{aligned}
$$

Как видно из формулы (27), математическое ожидание коэффициентов разложения $A_{m}$ зависит от компонентов корреляционной матрицы $\left\langle\tilde{a}_{j} \tilde{a}_{k}\right\rangle$, которые в свою очередь выражаются через корреляционную функцию коэффициента упругости основания по формуле (17). Таким образом, средние значения $\left\langle A_{m}\right\rangle$ и следовательно, математические ожидания разрешающей функции, перемещений и напряжений зависит от дисперсии коэффициента постели, характеризующей случайную неоднородность основания. Этим данная методика существенно отличается от метода малого параметра.

Заключение. Для коэффициента постели, изменяющегося в направлении продольной оси случайным образом, получено приближенное решение стохастической задачи на основе обобщенного метода Бубнова-Галеркина. Получена система уравнений относительно случайных коэффициентов разложения для моментов разрешающей функции. Выведены выражения для моментов коэффициентов разложения в предположении о гауссовском характере случайной функции коэффициента упругости основания.

\section{Список литературы}

1. Власов В.3. Тонкостенные пространственные системы. - М. : Госстройиздат, 1958. - 502 с.

2. Ибрагимов А. Расчет призматической замкнутой оболочки, свободно лежащий на неоднородном упругом основании. Рукопись представлена МИСИ им. В.В.Куйбышева. Деп. В ВНИИИС Госстроя СССр № 4147-83. - М. , 1983. $13 \mathrm{c}$.

3. Соболев Д.Н. Задача о штампе, вдавливаемом в статически неоднородном упругом основании. - Строительная механика и расчет сооружений, 1968, № 1, с. 1-4.

4. Власов В.З., Леонтьев Н.Н. Техническая теория расчета фундаментов на упругом основании. Тр. МИСИ , 1956, Сб. № 14, 12-31 с.

5. Ismayilov K. Critical stresses and deformations in a hinged-supported Rectangular plate beyond the elastic limi. European journal Of technical and Natural sciences № 3. Vienna, Austria. 2018.- 32-36. 
6. Ismayilov K. Critical strains and critical stresses in the steel rod beyond the Elastic limit. European science review. № 5-6. Vienna, Austria. 2018.-291-295.

7. Ismayilov K., Karimova K. Application of used automobile tires granules for road construction in Uzbekistan. Journal of Critical Reviews ISSN- 2394-5125 Vol 7, 946-948, Issue 12, 2020.

8. Ismayilov K., Karimova K. The Impact of Automobile Tires on the Environment from the Period of Raw Materials to the Disposal of Them International Journal of Recent Technology and Engineering (IJRTE) ISSN: 2277-3878, Volume-8 Issue-3, September 2019.

9. Mirsaidov MM, Sultanov TZ, Rumi DF 2013 Mag. Civ. Eng. 39(4) 94-105.

10. Indiaminov R., Butaev R., Ismayilov K., Isayev N., and A.Numonov Nonlinear integro-differential equations of bending of physically nonlinear viscoelastic plates. FORM-2020 IOP Conf. Series: Materials Science and Engineering 869 (2020) 052048 IOP Publishing doi:10.1088/1757$899 X / 869 / 5 / 052048$.

11. Ismayilov K 2018 Eur. J. Tech. Nat. Sci. Sci. J. 3 32-35.

12. T Mavlonov, BYuldoshev, Ismayilov K., and S.Toshev Nonlinear integrodifferential equations of bending of physically nonlinear viscoelastic plates. CONMECHYDRO - 2020 IOP Publishing IOP Conf. Series: Materials Science and Engineering 883 (2020) 012199 doi:10.1088/1757-99X/883/1/012199.

() Д.И.Кубаймуродов, 2021 